\title{
Determination of Cyanide and Thiocyanate in Sugihiratake Mushroom Using HPLC Method with Fluorometric Detection
}

\author{
Hiroshi Akiyama, ${ }^{* a}$ Toshihiko Toida, ${ }^{b}$ Shinobu Sakai, ${ }^{b}$ Yoshiaki Amakura, ${ }^{a}$ Kazunari Kondo, ${ }^{a}$ \\ Yoshiko Sugita-Konishi, ${ }^{a}$ and Tamio Maitani ${ }^{a}$ \\ ${ }^{a}$ National Institute of Health Sciences, 1-18-1, Kamiyoga, Setagaya-ku,Tokyo 158-8501, Japan and ${ }^{b}$ Graduate School of Pharmaceutical \\ Sciences, Chiba University, 1-33, Yayoi-cho, Inage-ku, Chiba 263-8522, Japan
}

(Received October 12, 2005; Accepted October 16, 2005; Published online October 19, 2005)

\begin{abstract}
A novel type of encephalopathy occurred in patients with chronic kidney diseases, which was associated with the ingestion of the Sugihiratake mushroom during the fall of 2004 in Japan. We attempted to investigate whether cyanide and thiocyanate are present in the Sugihiratake samples and determined the cyanide and thiocyanate levels in fifteen samples collected from different Japanese districts using HPLC with fluorometric detection. The cyanide ions and thiocyanate ions were detected in the ranges of N.D.-114.0 and N.D.-17.0 $\mu \mathrm{g} / \mathrm{g}$ in the samples, respectively. This is the first study to quantitatively detect cyanide and thiocyanate in the Sugihiratake mushrooms. This result demonstrated that cyanide exposure could occur from the intake of Sugihiratake mushrooms in one's diet. Furthermore, we discussed the possible association between cyanide and the onset of encephalopathy.
\end{abstract}

Key words — Sugihiratake, cyanide, thiocyanate, HPLC, encephalopathy

\section{INTRODUCTION}

Sugihiratake is the fungus Pleurocybella porrigens, which is a flat mushroom that grows on cedar and pine trees during the fall season, not only in the districts of northern Japan, but is also widely distributed in Japan. ${ }^{1)}$ It has a specific flavor, and many Japanese have been favorably consuming it in the processed foods of the highly popular miso (fermented bean paste soup) and the deep-fried food tempura. However, during the fall of 2004 in Japan,

\footnotetext{
*To whom correspondence should be addressed: National Institute of Health Sciences, 1-18-1, Kamiyoga, Setagaya-ku, Tokyo 158-8501 Japan. Tel.: +81-3-3700-9397; Fax: +81-33707-6950; E-mail: akiyama@nihs.go.jp
}

an outbreak of serious encephalopathy exclusively occurred in patients with chronic kidney diseases after the intake of this mushroom in many areas of Japan including the Akita, Yamagata, and Niigata Prefectures. Therefore, there have been some reports based on the clinical findings that encephalopathy was induced after the ingestion of this mushroom. The exact factors that induced the encephalopathy remain unclear and the association between the Sugihiratake mushroom intake and the onset of this novel type of encephalopathy is still currently controversial.

In the present study, we attempted to investigate the cyanide contents in wild Sugihiratake collected from several districts in Japan using a specific HPLC method with fluorometric detection, and were the first to detect cyanide in some of these samples. In addition, we discussed the possible association between cyanide intake and the onset of encephalopathy.

\section{MATERIALS AND METHODS}

Materials — The Sugihiratake mushroom samples were collected from the local health environment centers and the prefectural institutes of the public health and environmental science in Japan through the Ministry of Health, Labor and Welfare (MHLW) of Japan.

Reagents — A standard solution of potassium cyanide $(0.1 \mathrm{M})$ was prepared by dissolving potassium cyanide (Wako Pure Chemicals, Osaka, Japan) in $0.1 \mathrm{M}$ sodium hydroxide; the concentration of cyanide was calibrated by titration with silver nitrate using potassium iodide as the indicator according to the Liebig-Dènigès method. ${ }^{2,3)} \mathrm{A}$ standard solution of potassium thiocyanate (Wako Pure Chemicals) was prepared using redistilled water. All other 
Table 1. Cyanide and Thiocyanate Contents of the Sugihiratake Mushroom Samples

\begin{tabular}{clcc}
\hline \hline Sample No. & Producing district & $\begin{array}{c}\mathrm{CN}^{-} \\
(\mu \mathrm{g} / \mathrm{g} \text { dry weight })\end{array}$ & $\begin{array}{c}\mathrm{SCN}^{-} \\
(\mu \mathrm{g} / \mathrm{g} \text { dry weight })\end{array}$ \\
\hline 1 & Akita 1 & 12.7 & 1.1 \\
2 & Akita 2 & 25.5 & 4.6 \\
3 & Yamagata & 0.7 & 0.2 \\
4 & Niigata 1 & 1.8 & 0.5 \\
5 & Niigata 2 & 56.2 & 17.0 \\
6 & Mie & 3.1 & 1.6 \\
7 & Gifu 1 & 22.1 & 10.3 \\
8 & Gifu 2 & 114.0 & 9.4 \\
9 & Fukui 1 & 3.0 & 1.1 \\
10 & Fukui 2 & 0.9 & 1.4 \\
11 & Ishikawa & N.D. & 0.1 \\
12 & Kyoto & 1.2 & 0.2 \\
13 & Ibaraki & 96.6 & 8.4 \\
14 & Fukushima 1 & 0.6 & 0.1 \\
15 & Fukushima 2 & 0.3 & N.D. \\
\hline
\end{tabular}

N.D.: not detected.

chemicals were of analytical reagent grade.

Preparation of Sample Solution — The freezedried Sugihiratake sample was ground to a fine powder using a grinder (Retsch GmbH, Haan, Germany), and a $500 \mathrm{mg}$ test sample was extracted with $10.0 \mathrm{ml}$ of $0.1 \mathrm{M}$ sodium hydroxide by shaking overnight in a $50 \mathrm{ml}$ centrifuge tube. A one $\mathrm{ml}$ portion was then placed in the outer well of the Conway cell and $1.0 \mathrm{ml}$ of $0.1 \mathrm{M}$ sodium hydroxide was placed in the center chamber. The Conway cell and ground-glass cover were coated with silicone grease, and a glass cover was placed on top of the microdiffusion cell, leaving a small space for the addition of the acidic solution. To the samples described above for the determination of cyanide, $1.0 \mathrm{ml}$ of $1.2 \mathrm{M}$ sulfuric acid was added to the outer chamber. Subsequently, the ground-glass cover was moved to seal the microdiffusion cell. These cells were carefully rotated in order to mix the solution in the outer chamber. The cells were then rotated every $30 \mathrm{~min}$. Cyanide in the sample was allowed to diffuse for $4 \mathrm{hr}$ at room temperature and the liberated hydrogen cyanide was absorbed into the sodium hydroxide solution in the center chamber. An aliquot from the center chamber solution was analyzed by HPLC ${ }^{4)}$ For the recovery of thiocyanate from each sample during the pretreatment procedure, the Conway microdiffusion cell was kept for $24 \mathrm{hr}$ and the collected thiocyanate in the center chamber was analyzed by HPLC. Using the pretreatment procedure described above, the spiked standard cyanide and thiocyanate at the $1 \mu \mathrm{mol}$ level were recovered at $100.2 \pm 3.2$ and $95.4 \pm 5.5 \%$, respectively.

HPLC conditions ${ }^{4)}$ — The HPLC system consisted of a double-plunger pump (PU-1580, Jasco., Tokyo, Japan), an intelligent fluorescence detector (FP-920S, Jasco) with a xenon lamp and 12- $\mu$ l flow cell, a chromato-integrator (D-2500, Hitachi, Tokyo, Japan) and a sample injector (7725i, Reodyne, CA, U.S.A.). The HPLC conditions were as follows: column, a strong base anion exchange resin, TSK-Gel SAX $(150 \times 6 \mathrm{~mm}$ i.d., Tosoh Co., Tokyo, Japan $)$; eluent, $0.1 \mathrm{M}$ sodium acetate buffer ( $\mathrm{pH}$ 5.0) containing $0.2 \mathrm{M}$ sodium perchlorate (flow-rate, $1.0 \mathrm{ml} / \mathrm{min}$ ); chlorination reagent, $0.1 \%$ chloramines $\mathrm{T}$ aqueous solution (flow-rate, $0.5 \mathrm{ml} / \mathrm{min}$ ); pyridinebarbituric acid reagent, a mixture of barbituric acid $(1.5 \mathrm{~g})$, pyridine $(15 \mathrm{ml})$, concentrated hydrochloric acid $(3 \mathrm{ml})$ and redistilled water $(82 \mathrm{ml})$ (flow-rate, $0.5 \mathrm{ml} / \mathrm{min}$ ); the excitation and emission wavelengths of the detector were 583 and $607 \mathrm{~nm}$, respectively.

\section{RESULTS AND DISCUSSION}

We attempted to investigate the cyanide content in the Sugihiratake samples and determined the cyanide in fifteen samples collected from different Japanese districts using HPLC with fluorometric detection. As shown in Table 1, we detected the cyanide ions and thiocyanate ions in the ranges of N.D.- 


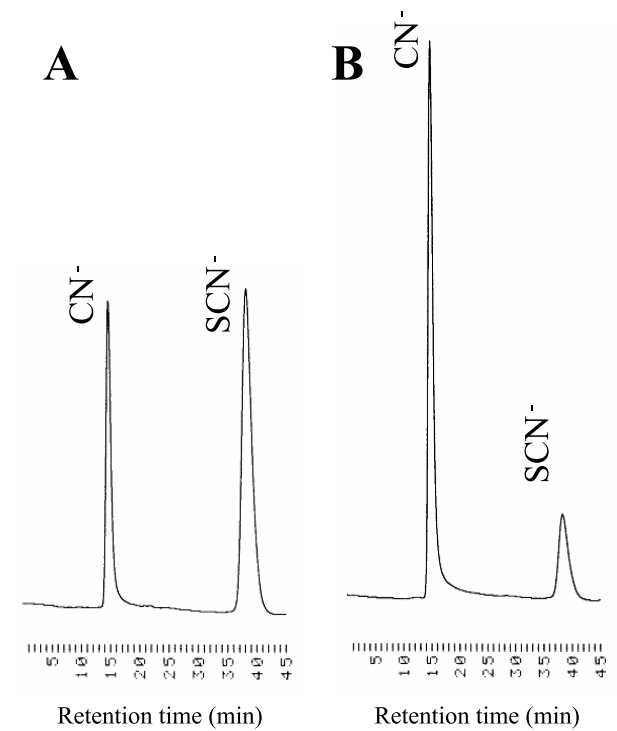

Fig. 1. Typical HPLC Chromatograms of Cyanide Ion and Thiocyanate Ion in a Sugihiratake Mushroom Sample

A; $\mathrm{CN}^{-}$and $\mathrm{SCN}^{-}$Standard (10 mM, respectively), B; Sample 5 (Niigata).

$114.0 \mu \mathrm{g} / \mathrm{g}$ dry weight and N.D. $-17.0 \mu \mathrm{g} / \mathrm{g}$ dry weight, respectively. These levels would not be lethal doses for acute toxicity even if $1 \mathrm{~kg}$ of the maximum level sample was consumed, because the lethal dose of cyanide is estimated to be $200-300 \mathrm{mg}$ for an adult human. This result demonstrates that cyanide and thiocyanite exposures would occur from the intake of Sugihiratake mushrooms.

As for the determination of cyanide, the conventional spectrophotometric method has been previously used in forensic toxicology and waste water regulation. ${ }^{5)}$ However, it is known that thiocyanate could cause a serious false positive using the conventional method. Therefore, even forensic scientists have often mistakenly estimated cyanide because of the false positive caused by thiocyanate. $\left.{ }^{6}\right)$ In the present study, we used the specifc and sensitive HPLC method of Toida et al. ${ }^{4)}$ after the pretreatment using the Conway cell. As shown in Fig. 1, we could simultaneously detect cyanide and thiocyanate using the HPLC system with an ion chromatographic column with non-interference determination. To our knowledge, this is the first report to accurately determine the content of cyanide and thiocyanate in Sugihiratake mushrooms. Since we confirmed the non-production of cyanide from linamarin, glucosidic cyanogens, using alkali solution (data not shown), we consider that the cyanide detected in the Sugihiratake samples would be present in the sodium form or potassium form.
Many food plants including agriculturally important species, such as cassava, flax, sorghum, alfalfa, peaches, almonds, and beans, are known to be cyanogenic. ${ }^{7)}$ Center African cassava flour contains sufficient quantities of cyanogens. When cassava is the staple part of the diet, the human daily consumption is equivalent to about one-half the lethal dose, which probably is thought to be the reason for the widespread and chronic neurological disorders called "konzo" found in this area. ${ }^{7)}$ In addition, the cyanide production has been observed in a wide range of fungi, such as Phaeolepiota aurea, Rozites caperatus, Leucopaxillus giganteus, and Pleurocybella porringens (Sugihiratake), ${ }^{8}$ although there are no reports to describe the cyanide content in these fungi. Some reports suggested that the cyanide production in fungi could be associated with snow mold disease and fairy ring disease in some plants. ${ }^{9)}$

To date, some clinical case studies involving the outbreak of acute encephalopathy that occurred in Japan during the fall of 2004 have already been reported. ${ }^{10-13)}$ All the cases were involved with the intake of Sugihiratake and the patients had varying degrees of renal dysfunction. The common clinical syndrome was characterized by weakness and involuntary movements of the extremities or dysarthria at the onset of the disease and subsequent intractable focal motor seizures, resulting in the generalized status of epilepticus or a comatose state. Some brain MRI examinations revealed that diffuse lesions in the basal ganglia and multiple ringed lesions in the cerebral cortex.

While there are some studies that cyanide could induce encephalopathy. ${ }^{14-19)}$ Smith et al. showed that comparatively small doses of cyanide given over long intervals can produce histological changes in the central nervous system of the rat. ${ }^{14)}$ As for the clinical study, Rachinger et al. showed that the toxicity of cyanide caused cerebral damage, primarily to the basal ganglia in the case report of patients that attempted suicide with cyanide. ${ }^{17)}$ This symptom appears to be consistent with those cases that occurred in the Akita Prefecture of Japan.

Furthermore, a recent study showed that cyanide and thiocyanate do accumulate in haemodialysis patients due to tobacco smoking. ${ }^{20)}$ Cyanide is known to be metabolized to thiocyanate by the enzyme rhodanese. This reaction is essential to life through its detoxification of cyanide, and thiocyanate synthesis can be accelerated under cyanide-loaded conditions such as tobacco smoking. ${ }^{21)}$ In addition, a 
recent study showed that the risk of cerebral infarction stroke was significantly increased in individuals having high serum thiocyanate concentrations. ${ }^{22}$ There also is some evidence that thiocyanate enhances the action of glutamate in a subclass of neuronal glutamate receptors which are involved in the neurodegenerative disorders. ${ }^{23)}$

These results suggested that the ingestion of cyanide from foods could also induce the accumulation of cyanide and thiocyanate in the blood of patients with chronic kidney diseases and might be associated with the onset of encephalopathy. However, since the human capacity for detoxification and the toxicity of cyanide and thiocyanate from food exposure in haemodialysis patients have not been fully investigated, a further investigation is necessary in order to elucidate the relationship between the cyanide in Sugihiratake mushrooms and the onset of a new type of encephalopathy that occurred in Japan during the fall of 2004. Nevertheless, it should be noted that there could still be other factors and other substances that caused this novel type of encephalopathy in addition to the ingestion of cyanide from Sugihiratake mushrooms.

In conclusion, we were the first to determine the cyanide content in the Sugihiratake samples collected from certain areas of Japan during the fall of 2004. In addition, we showed that some samples could contain cyanide in the range of N.D.$114.0 \mu \mathrm{g} / \mathrm{g}$, and suggested that its form in the Sugihiratake samples could be the sodium or potassium salt. This finding suggested that cyanide in Sugihiratake might be associated with the onset of a novel type of encephalopathy in patients with chronic kidney diseases, which occurred in Japan during the fall of 2004.

Acknowledgements We thank Dr. Taku Nagao and Dr. Yukihiro Goda for their useful suggestions. This study was supported by a grant from the Ministry of Health, Labour and Welfare of Japan in the fiscal year 2004.

\section{REFERENCES}

1) Gejyo, F., Homma, N., Higuchi, N., Ataka, K., Teramura, T., Alchi, B., Suzuki, Y., Nishi, S., Narita, I. and Japanese Society of Nephrology (2005) A novel type of encephalopathy associated with mushroom Sugihiratake ingestion in patients with chronic kidney diseases. Kidney Int., 68, 188-192.

2) Liebig, J. (1852) Process for determining the amount of hydrocyanic acid in medical prussic acid, bitter almond water, and laurel water. Q. J. Chem. Soc. Lond., 4, 219-221.

3) Bark, L. S. and Higson, H. G. (1963) A review of methods available for the detection and determination of small amounts of cyanide. Analyst (London), 88, 751-760.

4) Toida, T., Togawa, T., Tanabe, S. and Imanari, T. (1984) Determination of cyanide and thiocyanate in blood plasma and red cells by high-performance liquid chromatography with fluorometric detection. $J$. Chromatogr., 308, 133-141.

5) Boxer, G. E. and Rickards, J. C. (1952) Determination of thiocyanate in body fluids. Arch. Biochem. Biophys., 39, 292-300.

6) Seto, Y. (2002) False cyanide detection. Anal. Chem., 74, 135A-141A.

7) Eyjolfsson, R. (1970) Recent advances in the chemistry of cyanogenic glycosides. Fortscher. Chem. Org. Naturst., 28, 74-107.

8) Singer, R. (1975) Agaricales in Modern Taxonomy In Agaricales in Modern Taxonomy 3rd ed., J. Cramer, Hafner Publishing Co., New York, U.S.A. p. 912.

9) Knowles, C. J. (1976) Microorganisms and cyanide. Bacteriol. Rev., 40, 652-680.

10) Obara, K., Okawa, S., Kobayashi, M., Takahashi, S., Watanabe, S. and Toyoshima, I. (2005) A case of encephalitis-type encephalopathy related to Pleurocybella porrigens (Sugihiratake). Rinsho Shinkeigaku, 45, 253-256.

11) Kuwabara, T., Arai, A., Honma, N. and Nishizawa, M. (2005) Acute encephalopathy among patients with renal dysfunction after ingestion of "sugihiratake", angel's wing mushroom - study on the incipient cases in the northern area of Niigata Prefecture. Rinsho Shinkeigaku, 45, 239-245.

12) Kurokawa, K., Sato, H., Nakajima, K., Kawanami, T. and Kato, T. (2005) Clinical, neuroimaging and electroencephalographic findings of encephalopathy occuring after the ingestion of "sugihiratake" (Pleurocybella porrigens), an autumn mashroom: a report of two cases. Rinsho Shinkeigaku, 45, 111116.

13) Kato, T., Kawanami, T., Shimizu, H., Kurokawa, K., Sato, H., Nakajima, K., Nomoto, T., Seta, T., Kamei, T., Yoshino, H., Sasagawa, I., Ito, M., Karasawa, S., Kimura, H., Suzuki, Y., Degawa, N., Tagawa, A., Ataka, K., Ando, S., Omae, T. and Shikama, Y. (2004) An outbreak of encephalopathy after eating autumn mushroom (Sugihiratake; Pleurocybella porrigens) in patients with renal failure: a clinical 
analysis of ten cases in Yamagata, Japan. No To Shinkei, 56, 999-1007.

14) Smith, A. D. M., Duchkett, S. and Waters, A. H. (1963) Neuropathological changes in chronic cyanide intoxication. Nature (London), 200, 179-181.

15) Smith, A. D. M. (1964) Cyanide encephalopathy in man? Lancet, 26, 668-670.

16) Funata, N., Song, S. Y., Okeda, R., Funata, M. and Higashino, F. (1984) A study of experimental cyanide encephalopathy in the acute phase - physiological and neuropathological correlation. Acta Neuropathol. (Berl), 64, 99-107.

17) Rachinger, J., Fellner, F. A., Stieglbauer, K. and Trenkler, J. (2002) MR changes after acute cyanide intoxication. AJNR Am. J. Neuroradiol., 23, 13981401.

18) Yen, D., Tsai, J., Wang, L. M., Kao, W. F., Hu, S. C., Lee, C. H. and Deng, J. F. (1995) The clinical experience of acute cyanide poisoning. Am. J. Emerg. Med., 13, 524-528.

19) Wilson, J. (1983) Cyanide in human disease: a re- view of clinical and laboratory evidence. Fundam. Appl. Toxicol., 3, 397-399.

20) Hasuike, Y., Nakanishi, T., Moriguchi, R., Otaki, Y., Nanami, M., Hama, Y., Naka, M., Miyagawa, K., Izumi, M. and Takamitsu, Y. (2004) Accumulation of cyanide and thiocyanate in haemodialysis patients. Nephrol. Dial. Transplant., 19, 1474-1479.

21) Pettigrew, A. R. and Fell, G. S. (1972) Simplified colorimetric determination of thiocyanate in biological fluids, and its application to investigation of the toxic amblyopias. Clin. Chem., 18, 996-1000.

22) Wang, H., Sekine, M., Yokokawa, H., Hamanishi, S., Chen, X., Sayama, M., Naruse, Y., Nakagawa, H. and Kagamimori, S. (2001) The relationship between new stroke onset and serum thiocyanate as an indicator to cigarette smoking. J. Epidemiol., 11, 233-237.

23) Spencer, P. S. (1999) Food toxins, ampa receptors, and motor neuron diseases. Drug Metab. Rev., 31, 561-587. 